\title{
Emerging Applications of
}

\section{Synchrotron-Generated Microbeams in Neurological and Neurosurgical Research}

\author{
Pantaleo Romanelli ${ }^{1 *}$, Alberto Bravin ${ }^{2}$ and Giuseppe Battaglia ${ }^{3}$ \\ ${ }^{1}$ Cyberknife Center,Centro Diagnostico Italiano(CDI), Italy
}

${ }^{2}$ European Synchrotron Radiation Facility (ESRF), France

${ }^{3}$ IRCCS Neuromed, Italy

Submission: November 19, 2018; Published: November 28, 2018

*Corresponding author: Pantaleo Romanelli, Cyberknife Center, Centro Diagnostico Italiano (CDI), Milano, Italy

Keywords: Microbeams, Brain disorders, Tissue, Hippocampus, Neurologic deficit, Radiation, Spinal cord, Array, Radiosurgery, Modulation

Pathologic activity, Cortex, Application, Surgical techniques

\section{Mini Review}

Recent research performed at ESRF has shown that microbeams arrays delivering incident doses from 300 to 600 GY generate the equivalent of a microsurgical incision over the cortex and hippocampus. These microbeam incisions generate no neurological deficit when delivered to eloquent cortex, offering an attractive new way to treat seizures originating from brain regions not amenable to surgical resection and to modulate neural networks involved in a wide variety of functional brain disorders

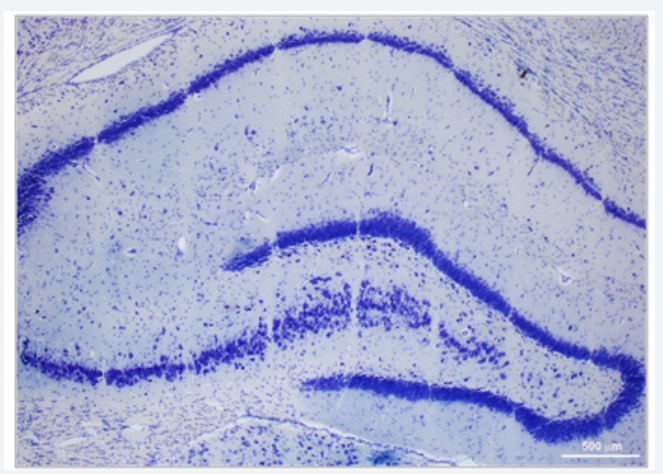

Figure 1: Nissl staining of the dorsal hippocampus underwent to synchrotron-generated hippocampal transactions, 11 months after delivery of an array of ten parallel microbeams to a rat brain. Microbeam size was $75 \mu \mathrm{m}$ and spacing across the beams was $400 \mu \mathrm{m}$.

The minimal beam divergence of synchrotron-generated micro planar beams (microbeams) is associated with an extremely sharp dose deposition along the beam path, allowing the delivery of hundreds to thousands of Gy to microscopic tissues slices without inducing radio necrosis [1]. Synchrotrongenerated microbeams can also be used to generate wholebrain volumetric imaging with details going down to subcellular structures [2]. This recently described microimaging modality, called phase-contrast computed tomography, generates extremely bright and detailed imaging enhancing the detection of microscopic cancer spread [2]. Microbeam treatment of solid tumors allows the delivery of doses up to 1000 Gy with selective damage to the tumor and preservation of healthy tissue [3]. This" tissue-sparing effect" is retained with beam sizes up to $0,68 \mathrm{~mm}$ : no neurologic deficit was induced by the irradiation of the spinal cord of healthy rats with a microbeam array made by four $0,68 \mathrm{~mm}$ parallel beams spaced $4 \mathrm{~mm}$ and delivering an incident dose of 400 Gy [4].

The ability of microbeams to avoid radio necrosis and to preserve the architecture of the irradiated tissue despite the very intense doses delivered is attributed to several factors, including the high radio resistance of normal large vessels to very high doses delivered in microbeams, the rapid regeneration of normal micro vessels and the spatial fractionation [4-6]. An array of parallel micro planar beams delivers a peak dose of several hundreds of Gy to a series of parallel short segments of the vascular bed while the adjacent vessels and tissue fall into the valley dose region receiving doses in the range of 1 to $4 \mathrm{~Gy}$. The rapid growth of uninjured capillaries widely spreading and bridging along the interfaces of peak and valley dose provides an immediate restoration of blood supply avoiding the development of radio necrosis. Figure 1 shows a microbeam array through the hippocampus, a structure very sensitive to conventional 
irradiation, which is well known to disrupt neurogenesis causing hippocampal atrophy and radio-induced dementia. An array of parallel beams (thickness 75 microns, inter-beam spacing 400 microns) delivering an incident dose of 600 Gy generated hippocampal transections without atrophy or distortion of the overall anatomy.

Hippocampal neurogenesis, typically disrupted by whole brain radiation, was also preserved. The wide spatial interface between the unhindered tissue in the valleys and the tissue receiving peak doses within the microbeam paths facilitated a widespread vascular recolonization of the necrotic regions, thus preventing the dissolution of the tissue architecture and the disruption of neurogenesis induced by delayed vascular effects. It appears that the neoplastic vasculature is unable to replicate this fast repair of the segments hit by the peak dose, facilitating the development of radio necrosis over the irradiated tumor [6].

The introduction of stereotactic techniques to deliver microbeam arrays focused over a selected target volume allows to deposit doses of several hundred Gy over the target while keeping the tissue sparing effect over the adjacent tissues. Microbeam Radio Surgery (MRS) is currently performed at the ESRF biomedical beamline, either by directing beams to the target in a convergent is centric fashion (creating a hot spot where the dose is enhanced by the overlapping of the beams) or interlacing 2-to-4 microbeam arrays over the target. Imageguided MRS has been used at ESRF to finely ablate selected volumes (such as the subthalamic nucleus, the substantia nigra and the caudate nucleus) in the rat brain.

These micro radio surgical lesions are useful to develop experimental models to study PD, Huntington's disease and many other CNS disorders. Modulation of brain networks with the aim to disrupt pathologic activity while saving neurological function is an innovative research field recently developed at ESRF. Microbeams arrays are placed over selected cortical areas in order hit tangentially and cut the horizontal axons connecting adjacent cortical columns, thus replicating the effects of microsurgical cortical transections, a procedure aiming to parcellize and disconnect an epileptic focus located in eloquent cortex. Cutting the horizontal axons required for the spreading of epileptic activity is an effective way to control seizures without inducing neurologic dysfunction, which would be necessarily associated to a respective or ablative procedure on eloquent cortex. Synchrotron-generated microbeams have been used to create transections over the sensorimotor cortex, the visual cortex and the hippocampus. Sensorimotor cortex microbeam transections generated fast abolition of status epilepticus induced by focal cortical injection of kainic acid while motor function was preserved. This novel experimental application of microbeams provides a new and attractive tool to modulate cortical function by transecting the fibers connecting the cortical columns while keeping intact the neurological functions sub served by the colums. Aside from the tight dosimetry, the low energy of monochromatic beams makes them well-suited to treat superficial targets such us the cortex [7-10].

Microbeam transections, either placed over neocortical seizure foci or through the hippocampus, could prove to be an excellent tool to be added to the current radio surgical techniques used to control seizures and their use can be extended to modulate the cortical functions in a wide variety of functional brain disorders.

\section{Conclusion}

Synchrotron-generated microbeams have been extensively characterized as a new experimental treatment for brain tumors. The irradiation of normal brain and spinal cord with microbeam arrays is characterized by a distinct tissue-sparing effect. Incident doses between 300 and 600 Gy are well tolerated by the CNS with no histological evidence of brain damage outside the beam paths. A recent development of microbeam research is the use of microbeam transections to modulate pathological activity over eloquent brain cortex through the selective cutting of horizontal axons connecting adjacent columns. Microbeam transections can be placed on the hippocampus as well, offering a precious new tool to treat drug-refractory epilepsy. Nissl staining of the dorsal hippocampus underwent to synchrotron-generated hippocampal transactions, 11 months after delivery of an array of ten parallel microbeams to a rat brain. Microbeam size was $75 \mu \mathrm{m}$ and spacing across the beams was $400 \mu \mathrm{m}$. Incident dose was $600 \mathrm{~Gy}$. The treatment was well tolerated, causing no radio necrosis, edema, or evident behavioral or cognitive deficits. The hippocampal architecture between the microbeam transections is well preserved.

\section{References}

1. Romanelli P, Bravin A (2011) Synchrotron-generated microbeam radiosurgery: a novel experimental approach to modulate brain function. Neurol Res 33(8): 825-831.

2. Barbone GE, Bravin A, Romanelli P, Mittone A, Bucci D, et al. (2018) Micro-imaging of brain cancer radiation therapy using phase-contrast computed tomography. Int J Rad Onc Biol Phys 101(4): 964-984.

3. Laissue JA, Geiser G, Spanne PO, Dilmanian FA, Gebbers JO, et al. (1998) Neuropathology of ablation of rat gliosarcomas and contiguous brain tissues using a microplanar beam of synchrotron-wiggler-generated $X$ rays. Int J Cancer 78(5): 654-660.

4. Dilmanian FA, Zhong Z, Bacarian T, Benveniste H, Romanelli P, et al. (2006) Interlaced x-ray microplanar beams: a radiosurgery approach with clinical potential. Proc Natl Acad Sci U S A 103(25): 9709-9714.

5. Serduc R, Christen T, Laissue J, Farion R, Bouchet A, et al. (2008) Brain tumor vessel response to synchrotron microbeam radiation therapy: a short-term in vivo study. Phys Med Biol 53(13): 3609-3622.

6. Miura M, Blattmann H, Bräuer-Krisch E, Bravin A, Hanson AL, et al. (2006) Radiosurgical palliation of aggressive murine SCCVII squamous cell carcinomas using synchrotron-generated X-ray microbeams. Br J Radiol 79(937): 71-75.

7. Fardone E, Bravin A, Conti A, Bräuer-Krisch E, Requardt H, et al. (2017) Rat sensorimotor cortex tolerance to parallel transections induced by synchrotron-generated X-ray microbeams. Sci Rep 7(1): 14290. 


\section{Open Access Journal of Neurology \& Neurosurgery}

8. Romanelli P, Fardone E, Bucci D, Battaglia G, Bräuer-Krisch E, et al. (2015) Microradiosurgical cortical transections generated by synchrotron radiation. Phys Med 31(6): 642-646.

9. Fardone E, Pouyatos B, Bräuer-Krisch E, Bartzsch S, Mathieu H, et al. (2018) Synchrotron-generated microbeams induce hippocampal transections in rats. Sci Rep 8(1): 184.

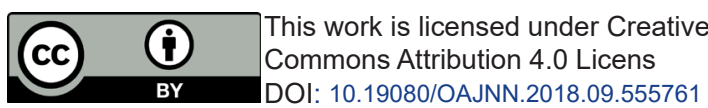

10. Romanelli P, Fardone E, Battaglia G, Bräuer-Krisch E, Prezado Y, et al. (2013) Synchrotron-generated microbeam sensorimotor cortex transections induce seizure control without disruption of neurological functions. PLoS One 8(1): e53549.

\section{Your next submission with Juniper Publishers} will reach you the below assets

- Quality Editorial service

- Swift Peer Review

- Reprints availability

- E-prints Service

- Manuscript Podcast for convenient understanding

- Global attainment for your research

- Manuscript accessibility in different formats

( Pdf, E-pub, Full Text, Audio)

- Unceasing customer service

Track the below URL for one-step submission https://juniperpublishers.com/online-submission.php 